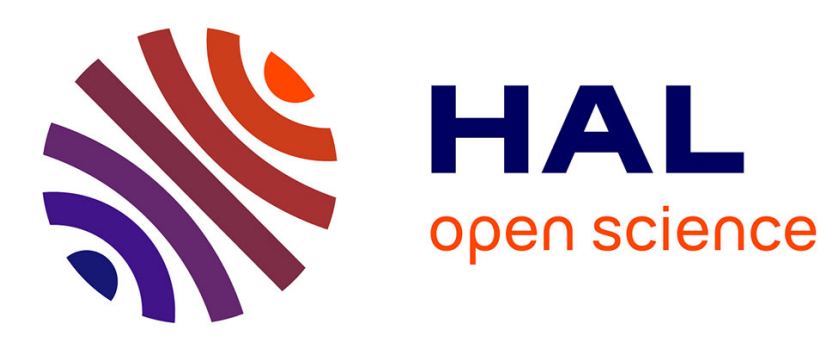

\title{
Spatial games and global optimization for the mobile association problem: the downlink case
}

Alonso Silva, Hamidou Tembine, Eitan Altman, Merouane Debbah

\section{To cite this version:}

Alonso Silva, Hamidou Tembine, Eitan Altman, Merouane Debbah. Spatial games and global optimization for the mobile association problem: the downlink case. 49th IEEE Conference on Decision and Control, Dec 2010, United States. 14 p. hal-00556197

\section{HAL Id: hal-00556197 \\ https://hal-centralesupelec.archives-ouvertes.fr/hal-00556197}

Submitted on 15 Jan 2011

HAL is a multi-disciplinary open access archive for the deposit and dissemination of scientific research documents, whether they are published or not. The documents may come from teaching and research institutions in France or abroad, or from public or private research centers.
L'archive ouverte pluridisciplinaire HAL, est destinée au dépôt et à la diffusion de documents scientifiques de niveau recherche, publiés ou non, émanant des établissements d'enseignement et de recherche français ou étrangers, des laboratoires publics ou privés. 


\title{
Spatial Games for the downlink case combining base station placement and mobile association
}

\author{
Alonso Silva*‡, Eitan Altman*, \\ Mérouane Debbahł Tembine Hamidouł
}

\begin{abstract}
We study the mobile association problem: we determine the cells corresponding to each base station, i.e, the locations at which intelligent mobile terminals prefer to connect to a given base station rather than to others. This paper proposes a new approach based on optimal transport theory to characterize the solution based on previous works on fluid approximations. We are able to characterize the global optimal solution, as well as the user optimal solution, for the downlink case problem.
\end{abstract}

\section{INTRODUCTION}

We consider the case where intelligent mobile terminals, capable of accessing multiple radio access technologies, will decide for themselves the wireless access technology to use and the access point to which to connect. Within this context, we study the mobile association problem, where we determine the locations at which intelligent mobile terminals prefer to connect to a given base station rather than to others.

For the user optimization problem, considering the interactions with other mobile terminals, starting from the seminal paper of Hotelling [1] a large area of research on location games has been developed. His paper [1] introduced the notion of spatial competition in a duopoly situation. Plastria [2] presented an overview of the research on locating one or more new facilities in an environment where competing facilities already exist. Gabszewicz and Thisse [3] provided another general overview on location games. Altman et al. [4] studied the duopoly situation in the uplink case in a line segment and realized that with the particular cost structure that arises in the cellular context more complex cells are obtained at equilibrium. Our work focuses on the downlink case and in a more general situation where a finite number of base stations can compete in a one-dimensional and two-dimensional case without any assumption on the symmetry of the users location. In order to do that, we propose a new framework for mobile association problems using optimal transport

\footnotetext{
*INRIA Sophia-Antipolis, France. Email: \{alonso.silva, eitan.altman\}@sophia.inria.fr

${ }^{\dagger}$ Alcatel-Lucent Chair in Flexible Radio - SUPELEC, France. Email: merouane.debbah@supelec.fr

‡Université d’Avignon, France. Email: tembine@ieee.org
} 
Table 1: Notation

\begin{tabular}{|c|l|}
\hline$N$ & Total number of MTs in the network \\
\hline$K$ & Total number of BSs \\
\hline$f$ & Deployment distribution of MTs \\
\hline$\left(x_{i}, y_{i}\right)$ & Position of the $i$-th BS \\
\hline$C_{i}$ & Cell determined by the $i$-th BS \\
\hline$N_{i}$ & Number of MTs associated to the $i$-th BS \\
\hline$M_{i}$ & Number of carriers offered by the $i$-th BS \\
\hline$\kappa_{i}$ & Penalization function of non-service \\
\hline$h_{i}$ & Channel gain function over the $i$-th cell \\
\hline$\xi_{i}$ & Path loss exponent over the $i$-th cell \\
\hline
\end{tabular}

theory (See [5] and references therein), a theory initiated by Monge [6] and Kantorovich [7] that has prove to be useful on many economical context $[8,9,10]$. There is a number of papers on "optimal transport" (see [11], and reference therein) however the authors in [11] consider an optimal selection of routes but do not use the rich theory of optimal transport. The works on stochastic geometry are similar to our analysis of wireless networks (see e.g. [12] and reference therein) but in our case we do not consider any particular deployment distribution function. Fluid models allow us to have this general deployment distribution function.

The remaining of this paper is organized as follows. Section 2 presents the formulation of the problem of minimizing the total power of the network under quality of service constraints. We address the problem for the downlink case where we considered two different policies: round robin scheduling policy (also known as time fair allocation policy) and rate fair allocation policy which are defined in Section 2 and studied precisely in Section 3 and Section 4 with uniform and nonhomogeneous distribution of users. In Section 5 we give numerical examples in both one-dimensional and two-dimensional mobile terminals distribution. Section 6 concludes the paper.

\section{The Model}

A summary of the notation used on this work can be found in Table 1. We consider a network deployed on a region, denoted by D, over the plane. The mobile terminals (MTs) are distributed according to a given deployment distribution function $f(x, y)$. To fix ideas, if the considered region is a square $\mathrm{D}=[0 \mathrm{~km}, 1 \mathrm{~km}] \times[0 \mathrm{~km}, 1 \mathrm{~km}]$ and the distribution of the users is uniform $f(x, y)=1$, 
then the proportion of users in the sub-region $\mathcal{A}=[0 \mathrm{~km}, 1 / 2 \mathrm{~km}] \times[0 \mathrm{~km}, 1 / 2 \mathrm{~km}]$ is

$$
\frac{\iint_{\mathcal{A}} f(x, y) d x d y}{\iint_{\mathcal{D}} f(x, y) d x d y}=\frac{\operatorname{Area}(\mathcal{A})}{\operatorname{Area}(\mathcal{D})}=1 / 4
$$

The first equality is obtained because the distribution of the users is uniform. However, the expression at the left-hand side is general and it is always equal to the proportion of mobiles in a sub-region $\mathcal{A}$. To simplify the notation, we scale the function $f$ such that

$$
\iint_{\mathcal{D}} f(x, y) d x d y=1
$$

Consequently, the function $f$ is a measure of the proportion of users over the network.

In the network, there are $K$ base stations (BSs), denoted by $\mathrm{BS}_{i}, i \in\{1, \ldots, K\}$, located at positions $\left(x_{i}, y_{i}\right), i \in\{1, \ldots, K\}$. We assume for the downlink case (transmission from BSs to MTs) that between neighboring BSs, they transmit in orthogonal channels (such as in OFDMA). Furthermore, we assume that the interference between BSs that are far from each other using the same sub-carrier is small. Consequently, instead of considering the SINR (Signal to Interference plus Noise Ratio), we consider as performance measure the SNR (Signal to Noise Ratio). We denote by $C_{i}$ the set of mobiles associated to the $i$-th BS and by $N_{i}$ the number of mobiles within that cell, both quantities to be determined. If the number of mobiles is greater than the maximum number of carriers available in the $i$-th cell, denoted by $\operatorname{Max}_{i}$, we consider a penalization cost function given by

$$
\kappa_{i}\left(N_{i}\right)=\left\{\begin{array}{cl}
0 & \text { if } N_{i} \leq \operatorname{Max}_{i} \\
\bar{\kappa}_{i}\left(N_{i}-\operatorname{Max}_{i}\right) & \text { if } N_{i}>\operatorname{Max}_{i}
\end{array}\right.
$$

We assume that $\bar{\kappa}_{i}$ can be either a constant or a non-decreasing function ${ }^{1}$. We first study the case $N_{i} \leq M$ and we study the general case in Section 3 .

The power transmitted from $\mathrm{BS}_{i}$ to a MT located at position $(x, y)$, is $P_{i}(x, y)$ and the power received at the MT is $P_{i}(x, y) h_{i}(x, y)$. We shall further assume that the channel gain corresponds to the path loss given by $h_{i}(x, y)=\left(\sqrt{R^{2}+d_{i}(x, y)^{2}}\right)^{-\xi}$ where $\xi$ is the path loss exponent, $R$ is the height of the base station, and $d_{i}(x, y)$ is the distance between a MT at position $(x, y)$ and $\mathrm{BS}_{i}$ located at $\left(x_{i}, y_{i}\right)$, i.e., $d_{i}(x, y)=\sqrt{\left(x_{i}-x\right)^{2}+\left(y_{i}-y\right)^{2}}$. The SNR received at mobile terminals at position $(x, y)$ in cell $C_{i}$ is given by $\operatorname{SNR}_{i}(x, y)=P_{i}(x, y) h_{i}(x, y) / \sigma^{2}$, where $\sigma^{2}$ is the noise power. We assume that the instantaneous mobile throughput is given by the following expression, which is based on Shannon's capacity theorem:

$$
\theta_{i}(x, y)=\log \left(1+\operatorname{SNR}_{i}(x, y)\right)
$$

We want to satisfy an average throughput for MTs located at position $(x, y)$ given by $\bar{\theta}(x, y)>0$. We shall consider for this objective two policies defined in [13]: (A) Round robin scheduling policy: under this policy, each BS devotes an equal fraction of time for transmission to each MT associated to it, and (B) Rate fair allocation policy: under this policy, each base station BS maintains a constant power sent to the mobile terminals within its cell. However, each base station modifies the fraction of time allowed to mobile terminals with different channel gains, in order that the average transmission rate demand is satisfied. For more information about this type of policies in the one dimensional case, see [13].

\footnotetext{
${ }^{1}$ For example, the maximum number of possible carriers in Wi-Max is around 2048, so by using this technology we have $\operatorname{Max}_{i}=2048$.
} 


\subsection{Round robin scheduling policy: Global Optimization}

Following this policy, $\mathrm{BS}_{i}$ devotes an equal fraction of time for transmission to MTs located within its cell $C_{i}$. The number of MTs located in the $i$-th cell is $N_{i}$. As $\mathrm{BS}_{i}$ divides its time of service proportional to the quantity of users within its cell, then the throughput following the round robin scheduling policy is given by $\theta_{i}^{\mathrm{RR}}(x, y)=\theta_{i}(x, y) / N_{i}$. In order to satisfy a throughput $\bar{\theta}(x, y)$ under this policy $\theta^{\mathrm{RR}}(x, y) \geq \bar{\theta}(x, y)$, or equivalently, in terms of the power

$$
P_{i}(x, y) \geq \frac{\sigma^{2}}{h_{i}(x, y)}\left(2^{N_{i} \bar{\theta}(x, y)}-1\right) .
$$

As our objective function is to minimize the total power of the network, the constraint will be reached, and we obtain

$$
P_{i}(x, y)=\sigma^{2}\left(2^{N_{i} \bar{\theta}(x, y)}-1\right)\left(\left[R^{2}+d_{i}^{2}(x, y)\right]^{1 / 2}\right)^{+\xi} .
$$

From last equation we can observe that: i) if the quantity of mobile terminals increases within the cell, it will need to transmit more power to each of the mobile terminals. The reason to do that is because the base station is dividing each time-slot into mini-slots with respect to the number of the mobiles within its cell, and ii) the function $\left(R^{2}+d_{i}^{2}(x, y)\right)^{\xi / 2}$ on the right hand side give us the dependence of the power with respect to the distance between the base station and the mobile terminal located at position $(x, y)$.

Our objective is to find the optimal mobile association in order to minimize the total power of the network. Then as the total power

$$
\begin{gathered}
P_{\text {total }}=\sum_{i=1}^{K} P_{i}^{\text {intra }} \\
\text { where } P_{i}^{\text {intra }}=\iint_{C_{i}} P_{i}(x, y) f(x, y) d x d y,
\end{gathered}
$$

$P_{i}^{\text {intra }}$ is the intracell power consumption in cell $C_{i}$.

The global optimization for the mobile association problem, that we denote $(\mathrm{RR})$, is to determine the cells $C_{i}, i \in\{1, \ldots, K\}$, to minimize the total power of the network:

$$
\text { Minimize } \sum_{i=1}^{K} \iint_{C_{i}} P_{i}(x, y) f(x, y) d x d y
$$

subject to (1), where $f(x, y)$ is the deployment distribution function of the users. We solve this problem in Section 3.

\subsection{Rate fair allocation policy: User Optimization}

In the rate fair allocation policy, each BS will maintain a constant power sent to MTs within its cell, i.e., $P_{i}(x, y)=P_{i}$ for each MT at location $(x, y)$ inside cell $C_{i}$. However, the BS modifies the 
fraction of time allotted to MTs, set in a way such that the average transmission rate to each MT with different channel gain is the same, denoted by $\Theta(x, y)$, for each mobile located at position $(x, y)$.

Let $r_{i}$ be the fixed rate of MTs located inside cell $C_{i}$. Following the rate fair allocation policy, the rate $r_{i}$ is given by (see e.g. [13]):

$$
r_{i}=\left(\iint_{C_{i}} \frac{1}{\log \left(1+\operatorname{SNR}_{i}(x, y)\right)} f(x, y) d x d y\right)^{-1} \beta .
$$

We seek for an equilibrium in the game in which each mobile terminal chooses from which base station is served and given the interactions with the other mobile terminals it doesn't have any incentive to change its strategy lonely. Similar notion of equilibrium has been studied in the context of large number of small players in road-traffic theory by Wardrop [14].

Definition.- The Wardrop equilibrium is given in the context of cellular systems by:

$$
\begin{gathered}
\text { If } \iint_{C_{i}} f(x, y) d x d y>0 \text {, then } \theta_{i}=\max _{1 \leq j \leq K} \theta_{j}\left(C_{j}\right), \\
\text { else if } \iint_{C_{i}} f(x, y) d x d y=0 \text {, then } \theta_{i} \leq \max _{1 \leq j \leq K} \theta_{j}\left(C_{j}\right) .
\end{gathered}
$$

A Wardrop equilibrium is the analog of a Nash equilibrium for the case of a large number of small players, where, in our case, we consider the mobile terminals as the players. In this setting, the Wardrop equilibrium indicates that if there is a positive proportion of mobile terminals associated to the $i$-th base station (the left-hand side condition in (2a)), then the throughput that the mobile terminals obtain is the maximum that they would obtain from any other base station (right-hand side consequence in (2a)). The second condition indicates that if there is one base station that doesn't have any mobile terminal associated to it (left-hand side condition in (2a)), it is because the mobile terminals can obtain more throughput by connecting with one of the other base stations (right-hand side consequence in $(2 \mathrm{a})$ ).

We assume that each base station is serving at least one mobile terminal, (if that is not the case, we remove the base station that is not serving any mobile terminal). Then, the equilibrium situation is given by

$$
\theta_{1}=\theta_{2}=\ldots=\theta_{K}
$$

To understand this equilibrium situation, consider as an example the simple case of two base stations: $\mathrm{BS}_{1}$ and $\mathrm{BS}_{2}$. Assume that $\mathrm{BS}_{1}$ offers more throughput than $\mathrm{BS}_{2}$. Then, the mobile terminals being served by $\mathrm{BS}_{2}$ will have an incentive to connect to $\mathrm{BS}_{1}$. The transmitted throughput depends inversely on the quantity of mobiles connected to the base station. As more mobile terminals try to connect to base station $\mathrm{BS}_{1}$ the throughput will diminish until arrive to the equilibrium where both base stations will offer the same throughput.

The condition $\theta_{1}=\theta_{2}=\ldots=\theta_{K}$ is equivalent in our setting to $r_{1}=r_{2}=\ldots=r_{K}$. Let us denote by $r$ to the rate offered by the base station at equilibrium, i.e., $r:=r_{1}=r_{2}=\ldots=r_{K}$. Then, as long as we are in the low-SNR regime, by using the low-SNR approximation of throughput, i.e., the throughput of a mobile at $\mathbf{x}$ is $\log (1+\mathrm{SNR}) \approx \mathrm{SNR}$, we have that:

$$
P_{i}\left(C_{i}\right)=\frac{r}{\Theta} \iint_{C_{i}} \sigma^{2}\left(R^{2}+d_{i}^{2}(x, y)\right)^{\xi / 2} f(x, y) d x d y
$$


We want to choose the optimal mobile assignment in order to minimize the total power of the network under the constraint that the mobile terminals have an average throughput of $\theta$, i.e., Minimize $_{C_{i}} P_{\text {total }}=\sum_{i=1}^{K} P_{i}^{\text {intra }}$

Then our problem reads

$$
(\mathrm{RF}) \quad \operatorname{Min}_{C_{i}} \sum_{i=1}^{K} \iint_{C_{i}} \sigma^{2}\left(R^{2}+d_{i}^{2}(x, y)\right)^{\xi / 2} f(x, y) d x d y .
$$

We will solve this problem in Section 4.

Thanks to optimal transport theory we are able to characterize the partitions on very general settings. For doing so, consider locations $\left(x_{1}, y_{1}\right) \ldots,\left(x_{K}, y_{K}\right)$, the Euclidean distance $d_{i}(x, y)=$ $\sqrt{\left(x-x_{i}\right)^{2}+\left(y-y_{i}\right)^{2}}$, and $F$ a continuous function.

Theorem 2.1. Consider the problem (P1)

$$
\operatorname{Min}_{C_{i}} \sum_{i=1}^{K} \iint_{C_{i}}\left[F\left(d_{i}(x, y)\right)+s_{i}\left(\iint_{C_{i}} f(\omega, z) d \omega d z\right)\right] f(x, y) d x d y
$$

where $C_{i}$ is the cell partition of $\mathrm{D}$. Suppose that $s_{i}$ are continuously differentiable, non-decreasing, and convex functions. The problem (P1) admits a solution that verifies

$$
(\mathrm{S} 1)\left\{\begin{aligned}
C_{i}= & \left\{x: F\left(d_{i}(x, y)\right)+s_{i}\left(N_{i}\right)+N_{i} \cdot s_{i}^{\prime}\left(N_{i}\right)\right. \\
& \left.\leq F\left(d_{j}(x, y)\right)+s_{j}\left(N_{j}\right)+N_{j} \cdot s_{j}^{\prime}\left(N_{j}\right)\right\} \\
N_{i}= & \iint_{C_{i}} f(\omega, z) d \omega d z
\end{aligned}\right.
$$

Proof.- See Appendix A

Theorem 2.2. Consider the problem (P2)

$$
\operatorname{Min}_{C_{i}} \sum_{i=1}^{K} \iint_{C_{i}}\left[F\left(d_{i}(x, y)\right) \cdot m_{i}\left(\iint_{C_{i}} f(\omega, z) d \omega d z\right)\right] f(x, y) d x d y
$$

where $C_{i}$ is the cell partition of $\mathrm{D}$. Suppose that $m_{i}$ are derivable. The problem (P2) admits a solution that verifies

$$
(\mathrm{S} 2)\left\{\begin{aligned}
C_{i}= & \left\{x: m_{i}\left(N_{i}\right) F\left(d_{i}(x, y)\right) f(x, y)+U_{i}(x, y)\right. \\
& \left.\leq m_{j}\left(N_{j}\right) F\left(d_{j}(x, y)\right) f(x, y)+U_{j}(x, y)\right\} \\
U_{i}= & m_{i}^{\prime}\left(N_{i}\right) \iint_{C_{i}} F\left(d_{i}(x, y)\right) f(x, y) d x d y \\
N_{i}= & \iint_{C_{i}} f(\omega, z) d \omega d z
\end{aligned}\right.
$$

Proof.- See Appendix B

Notice that in problem (P1) if the functions $s_{i} \equiv 0$ the solution of the system (S1) becomes the well known Voronoi cells. In problem (P2) if we have that the functions $h_{i} \equiv 1$ we find again the Voronoi cells. However in all the other cases the Voronoi configuration is not optimal. 


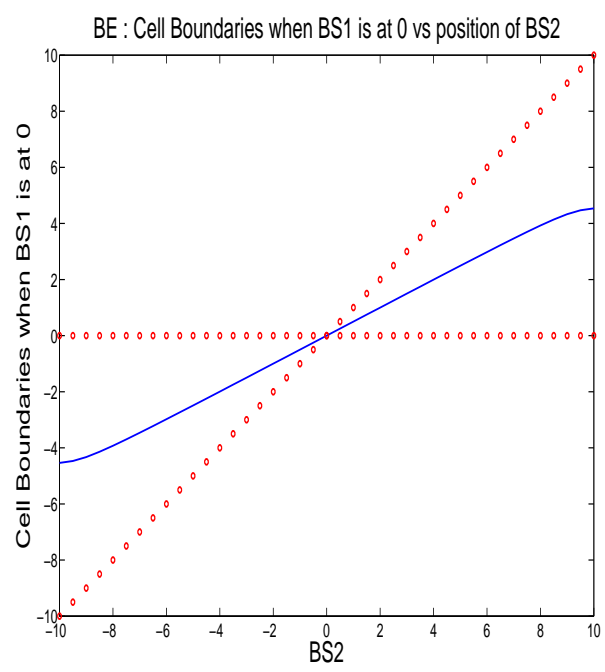

Figure 1: Wardrop Equilibrium: Thresholds determining the cell boundaries as a function of the location of the base stations. The network is deployed over the interval $[-10,10]$ (one-dimensional case) presented here vertically. We consider a uniform distribution of MTs and we find the threshold (blue line) determining the cell boundaries as a function of the base stations positions (red points) by changing the position of one of them. $\mathrm{BS}_{1}$ is fixed at position 0 and we change the position of $\mathrm{BS}_{2}$ from -10 to +10 .

\section{Round Robin Scheduling Policy}

We assume that a service provider wants to minimize the total power of the network while maintaining a certain average throughput of $\theta$ to each mobile terminal of the system using the round robin scheduling policy given by problem $(\mathrm{RR})$

$$
\operatorname{Min}_{C_{i}} \sum_{i=1}^{K} \iint_{C_{i}} \sigma^{2}\left(R^{2}+d_{i}(x, y)^{2}\right)^{\xi / 2}\left(2^{N_{i} \theta}-1\right) f(x, y) d x d y .
$$

We see that this problem is an optimal transportation problem (P1) with cost function given by

$$
\begin{gathered}
F\left(d_{i}(x, y)\right)=\sigma^{2}\left(R^{2}+d_{i}(x, y)^{2}\right)^{\xi / 2} \text { and } \\
m_{i}(x, y)=\left(2^{N_{i} \theta}-1\right) .
\end{gathered}
$$

Proposition.- There exist a unique optimum given by

$$
\begin{aligned}
C_{i}= & \left\{x_{0} \in \mathrm{D}: d_{i}\left(x_{0}, y_{0}\right)^{p}+h_{i}\left(N_{i}\right)+N_{i} h_{i}^{\prime}\left(N_{i}\right)\right. \\
& \left.\leq d_{j}\left(x_{0}, y_{0}\right)^{p}+k_{j}\left(N_{j}\right)+N_{j} k_{j}^{\prime}\left(N_{j}\right), \forall j \neq i\right\} \\
N_{i}= & \iint_{C_{i}} f\left(x_{0}, y_{0}\right) d x_{0} d y_{0}
\end{aligned}
$$

Let's see a direct application of our results: 
Example 3.1. Consider a network of $N=2500$ mobile terminals distributed according to $f(x)$ in $[0, L]$ (for example, with $L=5.6$ miles for WiMaX radius cell). We consider two base stations at position $\mathrm{BS}_{1}=0$ and $\mathrm{BS}_{2}=L$ and $R=1$. Then, the mobile association threshold (the boundary between both cells, i.e., the location at which the mobile terminals obtain the same throughput by connecting to any of both base stations) is reduced to find $x$ such that the following equality holds:

$$
\begin{gathered}
\left(2^{N_{1} \theta}-1\right)\left(1+x^{2}\right) f(x)+2^{N_{1} \theta} \theta \log 2\left[x+\frac{x^{3}}{3}\right]= \\
\left(2^{N_{2} \theta}-1\right)\left(1+(1-x)^{2}\right) f(x) \\
+2^{N_{2} \theta} \theta \log 2\left[\frac{4}{3}-2 x+x^{2}-\frac{x^{3}}{3}\right]
\end{gathered}
$$

This is a fixed point equation on $x$.

If the mobile terminals are distributed uniformly, the optimal solution is given by $C_{1}=[0,1 / 2 L$ ) and $C_{2}=[1 / 2 L, L]$, which is the solution that Voronoi cells would give us and the number of mobile terminals connected to each base station is given by

$$
N_{1}=N_{2}=1250 .
$$

However, if the deployment distribution of the mobile terminals is more concentrated near $\mathrm{BS}_{2}$ than $\mathrm{BS}_{1}$, consider for example $f(x)=2 x$, the optimal solution is given by $C_{1}=[0, q)$ and $C_{2}=[q, L]$ with $q=0.6027 L$ and

$$
N_{1}=908 \text { and } N_{2}=1592 .
$$

Notice that in the global optimization solution, the number of mobile terminals connected to $\mathrm{BS}_{1}$ is smaller that the number of mobile terminals connected to $\mathrm{BS}_{2}$. However, the cell size is bigger.

\section{Rate fair allocation policy}

In this framework we give the possibility to mobile terminals to connect to the base station they prefer in order to minimize their power cost function while maintaining an average throughput of $\theta$. This is the reason why we denote this type of network as hybrid network.

As we saw this problem is equivalent to

$$
\operatorname{Min}_{C_{i}} \sum_{i=1}^{K} \iint_{C_{i}} \sigma^{2}\left(R^{2}+d_{i}^{2}(x, y)\right)^{\xi / 2} f(x, y) d x d y .
$$

Notice that this problem is equivalent to $(\mathrm{P} 1)$ where the functions $s_{i} \equiv 1$ The problem has then a solution given by

Proposition.- There exist a unique optimum given by

$$
\begin{aligned}
C_{i}= & \left\{x \in \mathrm{D}: \sigma^{2}\left(R^{2}+d_{i}^{2}\left(x_{0}, y_{0}\right)\right)^{\xi / 2},\right. \\
& \left.\leq \sigma^{2}\left(R^{2}+d_{j}^{2}\left(x_{0}, y_{0}\right)\right)^{\xi / 2}, \forall j \neq i\right\} \\
N_{i}= & \iint_{C_{i}} f\left(x_{0}, y_{0}\right) d x_{0} d y_{0}
\end{aligned}
$$

which is the Voronoi cells. 


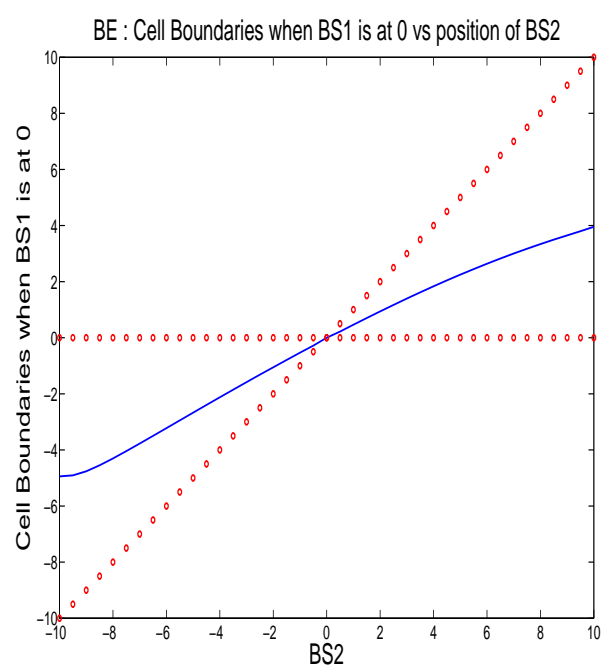

Figure 2: Wardrop Equilibrium in the Non-homogeneous case: The network is deployed over the interval $[-10,10]$ (one-dimensional case) presented here vertically. We find the threshold (blue line) determining the cell boundaries as a function of the base stations positions (red points) by changing the position of one of them. $\mathrm{BS}_{1}$ is fixed at position 0 and we change the position of $\mathrm{BS}_{2}$ from -10 to +10 . The deployment distribution of the MTs is given by $f(x)=(L-x) / 2 L^{2}$.

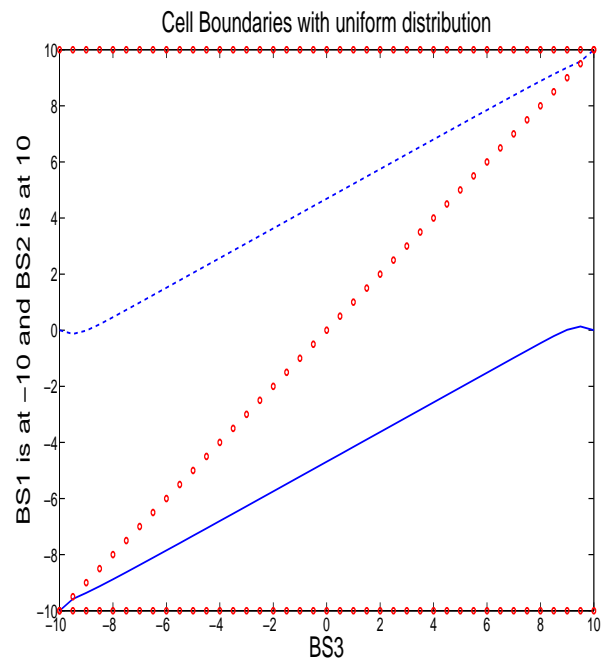

Figure 3: Wardrop Equilibrium with Multiple Base Stations: The grid area network is the interval $[-10,10]$ presented here vertically. We consider a uniform distribution of MTs. We find the threshold (blue line) determining the cell boundaries as a function of the base stations positions (red lines) by changing the position of one of them. $\mathrm{BS}_{1}$ is fixed at position -10 and $\mathrm{BS}_{2}$ is fixed at position 10 and we change the position of $\mathrm{BS}_{3}$ from -10 to +10 . 


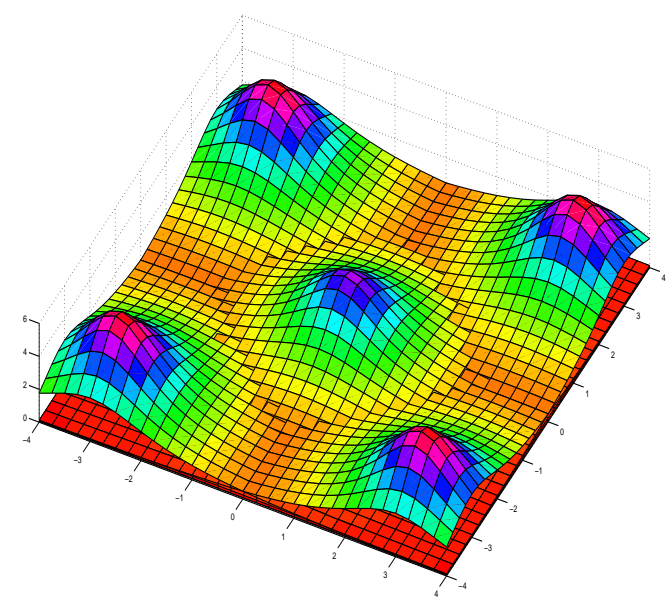

Figure 4: Wardrop Equilibrium in the 2D case: The grid area network is the square $[-4,4] \times[-4,4]$. We set the noise parameter $\sigma=0.3$ and we set four BSs at positions $\mathrm{BS}_{1}=(-3,-3) \mathrm{BS}_{2}=(3,-3)$ $\mathrm{BS}_{3}=(3,3) \mathrm{BS}_{4}=(-3,3)$ and one at the origin $\mathrm{BS}_{5}=(0,0)$. We determine the cell boundaries (deep lines) for the uniform distribution of users

\section{Validation of our theoretical model}

\subsection{One-dimensional case}

We first consider the one-dimensional case and we consider a uniform distribution of users in the interval $[-10,10]$. We set the noise parameter $\sigma=0.3$. In Fig. 1 , we fix one base station $\mathrm{BS}_{2}$ at position 0 and take as parameter the position of base station $\mathrm{BS}_{1}$. We consider the path loss exponent of $\xi=2$. Red lines shows the positions of the BSs. We are able to determine the cell boundary (solid blue curve) from $\mathrm{BS}_{1}$ and $\mathrm{BS}_{2}$ at different positions. In Fig. 3 we fix two base stations $\mathrm{BS}_{1}=-10$ and $\mathrm{BS}_{2}=10$ and we take as parameter the position of base station $\mathrm{BS}_{3}$. Red lines shows the positions of the BSs. We determine the cell boundary (solid blue curve) from $\mathrm{BS}_{1}$ and $\mathrm{BS}_{3}$ and the cell boundary (dashed blue curve) from $\mathrm{BS}_{2}$ and $\mathrm{BS}_{3}$.

\subsection{Two-dimensional case: Uniform and Non-Uniform distribution of users}

We consider the two-dimensional case. We consider the square $[-4,4] \times[-4,4]$ and the noise parameter $\sigma=0.3$. We set five base stations at positions $\mathrm{BS}_{1}=(-3,-3), \mathrm{BS}_{2}=(3,-3), \mathrm{BS}_{3}=(-3,3)$, $\mathrm{BS}_{4}=(3,3)$, and $\mathrm{BS}_{5}=(0,0)$. We determine the cell boundaries for the uniform distribution of MTs (see Fig. 4) and we compare it to the cell boundaries for the non-uniform distribution of MTs given by $f(x, y)=\left(L^{2}-\left(x^{2}+y^{2}\right)\right) / K$ where $K$ is a normalization factor. The latter situation can be interpreted as the situation when mobile terminals are more concentrated in the center and less concentrated in suburban areas as in Paris, New York or London. We observe that the cell size of 


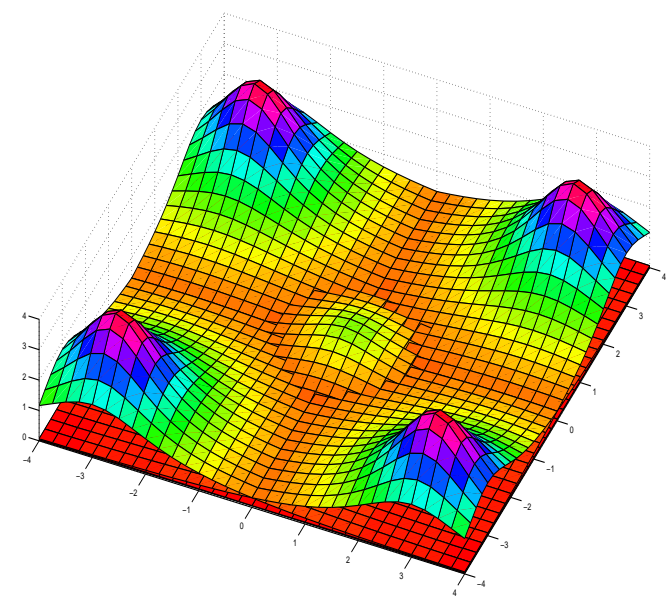

Figure 5: Wardrop Equilibrium in 2D Non-Homogeneous Case. The grid area network is the square $[-4,4] \times[-4,4]$. We set the noise parameter $\sigma=0.3$ and we set four BSs at positions $\mathrm{BS}_{1}=(-3,-3) \mathrm{BS}_{2}=(3,-3) \mathrm{BS}_{3}=(3,3) \mathrm{BS}_{4}=(-3,3)$ and one at the origin $\mathrm{BS}_{5}=(0,0)$. We determine the cell boundaries (deep lines) for the non-uniform distribution of users given by $f(x, y)=\left(L^{2}-\left(x^{2}+y^{2}\right)\right) / K$ where $K$ is a normalization factor. The latter situation takes into account when mobile terminals are more concentrated in the center and less concentrated in suburban areas.

the base station $\mathrm{BS}_{5}$ at the center is smaller than the others at the suburban areas. This can be explained by the fact that as the density of users is more concentrated in the center the interference is greater in the center than in the suburban areas and then the SINR is smaller in the center. However the quantity of users is greater than in the suburban areas.

\section{Conclusions}

We have studied a downlink mobile association game. We determined the location at which intelligent mobile terminals prefer to connect to a given base station rather than to others. Thanks to our proposed approach using optimal transport theory for mobile association we are able to completely characterize the mobile association and the cell formation under different policies from the mobile terminals point of view and as well as from the global system point of view.

\section{Appendix A}

Theorem 6.1. Consider the problem (P1)

$$
\operatorname{Min}_{C_{i}} \sum_{i=1}^{K} \iint_{C_{i}}\left[F\left(d_{i}(x, y)\right)+s_{i}\left(\iint_{C_{i}} f(\omega, z) d \omega d z\right)\right] f(x, y) d x d y
$$


where $C_{i}$ is the cell partition of D. Suppose that $s_{i}$ are continuously differentiable, non-decreasing, and convex functions. The problem (P1) admits a solution that verifies

$$
(\mathrm{S} 1)\left\{\begin{aligned}
C_{i}= & \left\{x: F\left(d_{i}(x, y)\right)+s_{i}\left(N_{i}\right)+N_{i} \cdot s_{i}^{\prime}\left(N_{i}\right)\right. \\
& \left.\leq F\left(d_{j}(x, y)\right)+s_{j}\left(N_{j}\right)+N_{j} \cdot s_{j}^{\prime}\left(N_{j}\right)\right\} \\
N_{i}= & \iint_{C_{i}} f(\omega, z) d \omega d z
\end{aligned}\right.
$$

Proof.- The proof is based mainly from Proposition 3.5 of Crippa et al. [15]. We first define a slightly more general relaxed formulation of the Monge problem:

$$
W_{F}(\mu, \nu):=\inf \left\{\left(\int_{\mathrm{D} \times \mathrm{D}} F\left(d_{i}(x, y)\right) d \gamma(x, y)\right): \gamma \in \Pi(\mu, \nu)\right\}
$$

where $\mu$ and $\nu$ are probability measures, and $\Pi(\mu, \nu)$ is the set of probability measures such that $\pi_{1}(\gamma)=\mu$ and $\pi_{2}(\gamma)=\nu$ where $\pi_{1}$ is the projection on the first component and $\pi_{2}$ is the projection on the second component.

We define also the unit simplex in $\mathbb{R}^{k}$ :

$$
S=\left\{c=\left(c_{1}, \ldots, c_{k}\right) \in \mathbb{R}^{k}: c_{i} \geq 0, \sum_{i=1}^{k} c_{i}=1\right\}
$$

The following step is to prove that

$$
\begin{gathered}
\operatorname{Min}_{C_{i}} \sum_{i=1}^{K} \iint_{C_{i}}\left[F\left(d_{i}(x, y)\right)+s_{i}\left(\iint_{C_{i}} f(\omega, z) d \omega d z\right)\right] f(x, y) d x d y \\
=\inf _{c \in S}\left\{W_{F}\left(f, \sum_{i=1}^{k} c_{i} \delta_{x_{i}}\right)+\sum_{i=1}^{k} s_{i}\left(c_{i}\right) c_{i}\right\}
\end{gathered}
$$

But this holds by Theorem 2.1 and by Remark 2.2 of [15].

In Proposition 3.5 by replacing $\left|x-x_{j}\right|$ by $F\left(d_{j}(x, y)\right)$ we obtain the thesis.

Then we check that this partition is an optimum.

\section{Appendix B}

Theorem 6.2. Consider the problem (P2)

$$
\operatorname{Min}_{C_{i}} \sum_{i=1}^{K} \iint_{C_{i}}\left[F\left(d_{i}(x, y)\right) \cdot m_{i}\left(\iint_{C_{i}} f(\omega, z) d \omega d z\right)\right] f(x, y) d x d y
$$


where $C_{i}$ is the cell partition of $\mathrm{D}$. Suppose that $m_{i}$ are derivable. The problem (P2) admits a solution that verifies

$$
(\mathrm{S} 2)\left\{\begin{aligned}
C_{i}= & \left\{x: m_{i}\left(N_{i}\right) F\left(d_{i}(x, y)\right) f(x, y)+U_{i}(x, y)\right. \\
& \left.\leq m_{j}\left(N_{j}\right) F\left(d_{j}(x, y)\right) f(x, y)+U_{j}(x, y)\right\} \\
U_{i}= & m_{i}^{\prime}\left(N_{i}\right) \iint_{C_{i}} F\left(d_{i}(x, y)\right) f(x, y) d x d y \\
N_{i}= & \iint_{C_{i}} f(\omega, z) d \omega d z .
\end{aligned}\right.
$$

Proof.- The proof is similar to Appendix A.

\section{References}

[1] H. Hotelling, "Stability in competition," The Economic Journal, vol. 39, no. 153, pp. 41-57, 1929. [Online]. Available: http://www.jstor.org/stable/2224214, Last visited: April 2010.

[2] F. Plastria, "Static competitive facility location: An overview of optimisation approaches," European Journal of Operational Research, vol. 129, no. 3, pp. 461 - 470, 2001. [Online]. Available: http://www.sciencedirect.com/science/article/B6VCT-421TKPJ1/2/47c0a2087d9d22e960343c3d931156b7, Last visited: April 2010.

[3] J. J. Gabszewicz and J.-F. Thisse, "Location," in Handbook of Game Theory with Economic Applications, ser. Handbook of Game Theory with Economic Applications, R. Aumann and S. Hart, Eds. Elsevier, 1992, vol. 1, ch. 9, pp. 281-304. [Online]. Available: http://ideas.repec.org/h/eee/gamchp/1-09.html, Last visited: April 2010.

[4] E. Altman, A. Kumar, C. K. Singh, and R. Sundaresan, "Spatial SINR games combining base station placement and mobile association," in INFOCOM. IEEE, 2009, pp. 16291637. [Online]. Available: http://dx.doi.org/10.1109/INFCOM.2009.5062081, Last visited: April 2010.

[5] C. Villani, Optimal transport, ser. Grundlehren der Mathematischen Wissenschaften [Fundamental Principles of Mathematical Sciences]. Berlin: Springer-Verlag, 2009, vol. 338, old and new. [Online]. Available: http://dx.doi.org/10.1007/978-3-540-71050-9, Last visited: April 2010.

[6] G. Monge, "Mémoire sur la théorie des déblais et des remblais," Histoire de l'Académie Royale des Sciences, 1871.

[7] L. V. Kantorovich, "On the transfer of masses," Dokl. Akad. Nauk., vol. 37, no. 2, pp. 227-229, 1942, translated in Management Science, Vol. 5, pp. 1-4, 1959.

[8] G. Buttazzo and F. Santambrogio, "A model for the optimal planning of an urban area," SIAM J. Math. Anal., vol. 37, no. 2, pp. 514-530 (electronic), 2005. [Online]. Available: http://dx.doi.org/10.1137/S0036141003438313, Last visited: April 2010. 
[9] G. Carlier and I. Ekeland, "The structure of cities," J. Global Optim., vol. 29, no. 4, pp. 371-376, 2004. [Online]. Available: http://dx.doi.org/10.1023/B:JOGO.0000047909.02031.ab, Last visited: April 2010.

[10] — - "Equilibrium structure of a bidimensional asymmetric city," Nonlinear Anal. Real World Appl., vol. 8, no. 3, pp. 725-748, 2007. [Online]. Available: http://dx.doi.org/10.1016/j.nonrwa.2006.02.008, Last visited: April 2010.

[11] Y. Yu, B. Danila, J. A. Marsh, and K. E. Bassler, "Optimal transport on wireless networks," Mar. 28 2007, comment: 5 pages, 4 figures. [Online]. Available: http://arxiv.org/abs/physics/0703261, Last visited: April 2010.

[12] F. Baccelli and B. Blaszczyszyn, Stochastic Geometry and Wireless Networks, Volume I Theory, ser. Foundations and Trends in Networking Vol. 3: No 3-4, pp 249-449, F. Baccelli and B. Blaszczyszyn, Eds. NoW Publishers, 2009, vol. 1, Stochastic Geometry and Wireless Networks, Volume II - Applications; see http://hal.inria.fr/inria-00403040. [Online]. Available: http://dx.doi.org/10.1561/1300000006 http://hal.inria.fr/inria-00403039/en/, Last visited: April 2010.

[13] G. S. Kasbekar, E. Altman, and S. Sarkar, "A hierarchical spatial game over licenced resources," in GameNets'09: Proceedings of the First ICST international conference on Game Theory for Networks. Piscataway, NJ, USA: IEEE Press, 2009, pp. 70-79.

[14] J. Wardrop, "Some theoretical aspects of road traffic research," Proceedings of the Institution of Civil Engineers, Part II, vol. 1, no. 36, pp. 352-362, 1952.

[15] G. Crippa, C. Jimenez, and A. Pratelli, "Optimum and equilibrium in a transport problem with queue penalization effect," Adv. Calc. Var., vol. 2, no. 3, pp. 207-246, 2009. [Online]. Available: http://dx.doi.org/10.1515/ACV.2009.009, Last visited: April 2010. 